\title{
Heart rate and respiratory rhythm dynamics on ascent to high altitude
}

Lewis A Lipsitz, Fred Hashimoto, Lawrence P Lubowsky, Joseph Mietus, George B Moody, Otto Appenzeller, Ary L Goldberger

\begin{abstract}
Objective-To investigate the alterations in autonomic control of heart rate at high altitude and to test the hypothesis that hypoxaemic stress during exposure to high altitude induces non-linear, periodic heart rate oscillations, similar to those seen in heart failure and the sleep apnoea syndrome.
\end{abstract}

Subjects-11 healthy subjects aged 24-64. Main outcome measures-24 hour ambulatory electrocardiogram records obtained at baseline $(1524 \mathrm{~m})$ and at $4700 \mathrm{~m}$. Simultaneous heart rate and respiratory dynamics during 2.5 hours of sleep by fast Fourier transform analysis of beat to beat heart rate and of an electrocardiographically derived respiration signal.

Results-All subjects had resting hypoxaemia at high altitude, with an average oxyhaemoglobin saturation of $81 \%(5 \%)$. There was no significant change in mean heart rate, but low frequency $(0.01-0.05$ Hz) spectral power was increased ( $P<$ $0.01)$ at high altitude. Time series analysis showed a complex range of non-linear sinus rhythm dynamics. Striking low frequency $(0.04-0.06 \mathrm{~Hz})$ heart rate oscillations were observed during sleep in eight subjects at high altitude. Analysis of the electrocardiographically derived respiration signal indicated that these heart rate oscillations correlated with low frequency respiratory oscillations.

Conclusions-These data suggest $(a)$ that increased low frequency power during high altitude exposure is not simply attributable to increased sympathetic modulation of heart rate, but relates to distinctive cardiopulmonary oscillations at $\sim 0.05 \mathrm{~Hz}$ and $(b)$ that the emergence of periodic heart rate oscillations at high altitude is consistent with an unstable cardiopulmonary control system that may develop on acute exposure to hypoxaemic stress.

\section{(Br Heart F 1995;74:390-396)}

Keywords: heart rate; respiratory rhythm; high altitude Marathon Clinics

Research Foundation Albuquerque, New

Mexico, USA

O Appenzeller

Correspondence to: Dr L A Lipsitz, Hebrew

Rehabilitation Center for

Aged Research and Training

Institute, 1200 Centre Street,

Roslindale MA 02131, USA.

Accepted for publication

7 March 1995 Perturbation of the non-linear physiological mechanisms regulating cardiopulmonary function may result in the emergence of highly periodic behaviour in heart rate and ventilatory dynamics. ${ }^{1-3}$ Perhaps the best described examples of such pathological oscillations are Cheyne-Stokes breathing in heart failure and the obstructive sleep apnoea syndrome. Each of these conditions is associated not only with periodic ventilatory dynamics but also with cyclic alterations in heart rate. ${ }^{4-6} \mathrm{We}$ hypothesised that hypoxaemic stress during exposure to high altitude would also induce non-linear, periodic heart rate dynamics.

\section{Subjects and methods}

We studied 11 healthy subjects (nine men and two women) aged 24 to 64 years. All were physically well trained in long distance running, but they were not accustomed to mountain climbing. Two lived at sea level and nine lived at $1524 \mathrm{~m}$. Twenty four hour ambulatory electrocardiograms were recorded with CardioData Dura-Lite cassette recorders (Model 2011) at low $(1524 \mathrm{~m})$ and high (4000-4700 m) altitude.

The lower altitude ambulatory electrocardiographic recordings were conducted during a usual work day and overnight sleep at $1524 \mathrm{~m}$. The high altitude recordings were performed during an expedition in the Himalayas at $4700 \mathrm{~m}$. Electrodes from the ambulatory monitors were attached to each subject during the first day at Mount Khachenjunga base camp $(4700 \mathrm{~m})$ after a three day trek ascending $3400 \mathrm{~m}$. The electrocardiographic recording was obtained over 24 hours and was divided into three time and activity segments $(a)$ eight hours of quiet activity from 1400 to $2200,(b)$ eight hours of sleep from 2200 to 0600 , and $(c)$ eight hours of extreme exertion from 0600 to 1400 . All subjects had resting oxyhaemoglobin saturation measurements obtained during the first day at base camp (4700 m only), using a Finapres oxygen saturation monitor (model 2300, Ohmeda, Louisville, Colorado, USA).

Tape recorded electrocardiographic signals were digitised at $128 \mathrm{~Hz}$ using a Marquette Series $\mathbf{8 0 0 0}$ Holter analysis system (Marquette Electronics, Milwaukee, Wisconsin, USA). The digitised data were then annotated according to an automated arrhythmia detection algorithm, and the beat annotations were verified by visual inspection. Cardiac rhythm analysis has been described previously in these subjects, who showed only infrequent ectopic activity.

Spectral analysis of sinus rhythm heart rate

was performed for sequential 15 minute sections of each 24 hour period according to the standard fast Fourier transform technique. Total heart rate power, a measure of overall heart rate variability, was computed for the 
Mean (SD) oxygenation and heart rate measurements at low and high altitude

\begin{tabular}{lll}
\hline & Low altitude $(1524 \mathrm{~m})$ & High altitude $(4700 \mathrm{~m})$ \\
\hline Resting Oxyhaemoglobin saturation (\%) & N/A & $81(5)$ \\
Heart rate during sleep (beats/minute) & $60(12)$ & $65(16)$ \\
Heart rate power during sleep & & \\
Total (0-0.50 Hz) & $21(12)$ & $38(14)^{\star}$ \\
Low frequency $(0 \cdot 01-0.15 \mathrm{~Hz})$ & $11(6)$ & $18(7)^{\star}$ \\
High frequency $(0 \cdot 15-0.50 \mathrm{~Hz})$ & $1.4(1.0)$ & $1.6(0.6)$ \\
\hline
\end{tabular}

N/A, not available.

$\star \mathrm{P}<0.01$.
Fourier transform analysis was also computed for continuous segments of the electrocardiographically derived respiration signal to compare the frequencies of respiratory and heart rate oscillations.

Changes in total, high, and low frequency heart rate power from low to high altitude were analysed by means of paired $t$ tests. Heart rate power is expressed in arbitrary units. All data are presented as means (SD). Statistical significance is defined as $\mathrm{P}<0.05$.

$0-0.50 \mathrm{~Hz}$ band. Relatively low frequer heart rate power was computed for the $0 \cdot 01-0.15 \mathrm{~Hz}$ band and high frequency heart rate power for the $0 \cdot 15-0.50 \mathrm{~Hz}$ band.

The heart rate time series and spectral plots were carefully examined by the investigators for evidence of non-linear dynamics, including oscillatory bursts and sudden changes in the frequency of oscillations (bifurcations). ${ }^{1-3}$ Episodes of low frequency heart rate oscillations $(0.04-0.06 \mathrm{~Hz})$ lasting 15 minutes or longer were identified by two observers, who independently examined each subject's 24 hour heart rate time series from low and high altitude.

To determine the relation between heart rate and respiratory oscillations, we derived a respiratory signal from the body surface electrocardiographic recording by measuring fluctuations in the mean cardiac electrical axis that accompany respiration. ${ }^{9}$ This technique is based on the observation that the body surface electrocardiogram is influenced by electrode motion relative to the heart and by changes in thoracic electrical impedance as the lungs fill and empty. By interpolating between axis measurements for each normal QRS interval a continuous electrocardiographically derived respiration signal is obtained. The relation between this signal and respiration has been confirmed by comparing the changes in axis direction with simultaneous measurements of chest circumference taken with a mercury strain gauge or pneumatic respiration transducer. ${ }^{9}$ In a small sample of patients with and without sleep apnoea on polysomnography the electrocardiographically derived respiration technique had a sensitivity of $75 \%$ for detecting apnoeic episodes, and gave no false positive results among 10 normal subjects without sleep apnoea. ${ }^{10}$ This method has also been used to examine the effect of respiratory rate and amplitude on heart rate variability in patients with congestive heart failure. ${ }^{5}$ Although noisy signals may limit the sensitivity of this method, the electrocardiographically derived respiration technique was particularly well suited for the measurement of respiratory rate in the field when lightweight portable monitors for both heart rate and respiration were not available. Since we did not aim to measure precisely ventilatory dynamics at high altitude, the electrocardiographically derived respiration signal provided a practical means to determine whether low frequency heart rate oscillations were associated with respiration.

Each 24 hour electrocardiographically derived respiration signal was scanned visually for evidence of abnormal breathing periodicities (see examples in figures 1 and 2). A fast

\section{Results}

All subjects had evidence of hypoxaemia while resting at high altitude, with an average oxyhaemoglobin saturation of $81 \%(5 \%)$ (table). None of the subjects had any signs or symptoms of high altitude pulmonary oedema.

Heart rate time series showed many different types of complicated oscillatory dynamics at various times of day. Examples of these dynamics are shown in figure 1 and include bursting activity, abrupt changes in frequency, spike-like oscillations, flutter-like (saw tooth) oscillations, and more complex oscillatory patterns. As these could represent non-stationarities resulting from different activities, environmental conditions, or measurement artifacts, we limited the analysis to comparable 2.5 hour periods of sleep at low and high altitude while all subjects were under similar steady state conditions. During these periods of sleep at high altitude there were striking low frequency heart rate oscillations $(0.04$ to 0.06 $\mathrm{Hz}$ ) with relatively abrupt onset and cessation in eight of the 11 subjects (figures 1 and 2). The peak to trough amplitude of these oscillations ranged from 12-40 beats/minute. Similar low frequency heart rate oscillations occurred at lower altitude in only two subjects (aged 61 and 44) during sleep. The presence or absence of low frequency oscillations was not related to the age, sex, or other identifiable characteristics of the subjects. There was no significant change in mean heart rate with altitude, but there was a significant increase in low and total heart rate spectral power during sleep at high altitude $(P<0.01$, table).

Analysis of the electrocardiographically derived respiration signal indicated that in seven out of eight subjects with low frequency heart rate oscillations, these oscillations were associated with alternating periods of apnoea and hyperpnoea at the same frequency (figures 2 and 3). Evidence of this breathing pattern from the electrocardiographically derived respiration signal was not noted in the absence of low frequency heart rate oscillations. Cross spectrum and coherence plots derived from the power spectra ${ }^{11}$ (figure 4) confirmed that the heart rate and electrocardiographically derived respiration signals oscillate at the same dominant frequency $(0.04-0.06 \mathrm{~Hz})$ and have a constant phase relation.

Figure 3 shows details of the relation between heart rate and respiratory oscillations in one subject. The oscillations were characterised by periods of hyperpnoea alternating with apnoea at a cycle length of approximately 


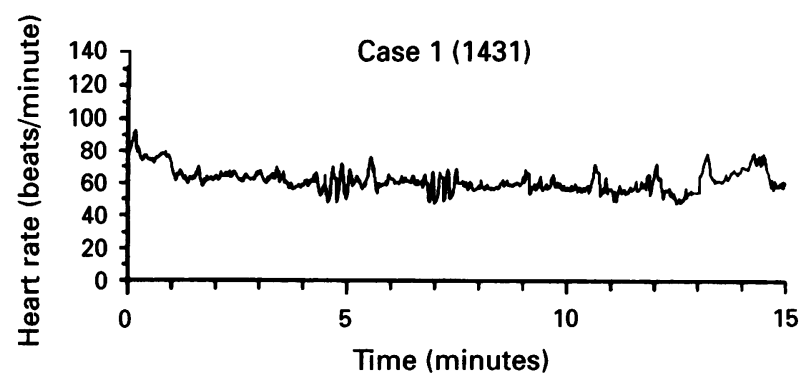

Bursting

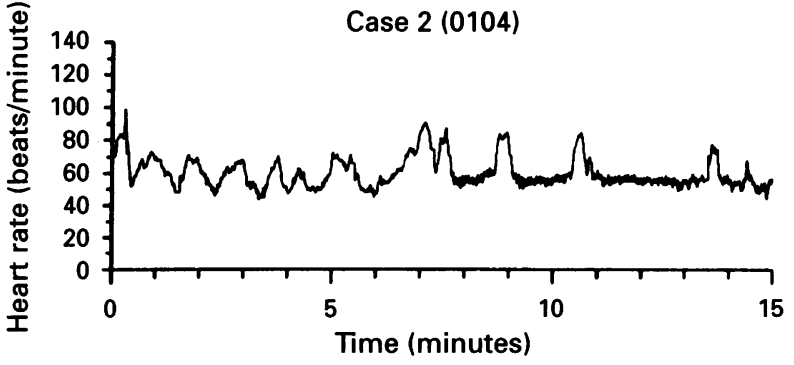

Abrupt changes
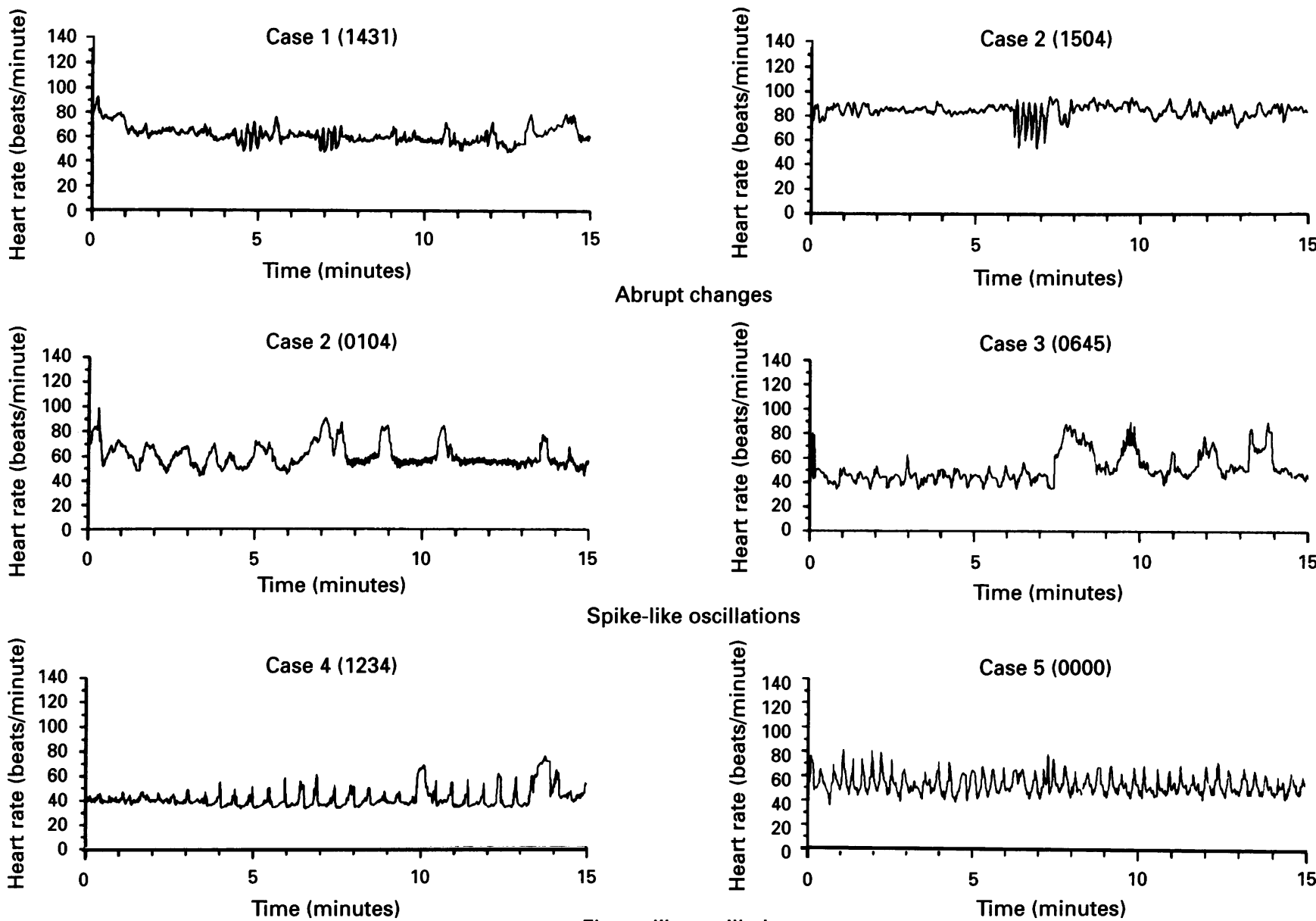

Spike-like oscillations

$20 \mathrm{~s}$. There was a consistent phase lag between the respiratory and heart rate cycles, such that periods of hyperpnoea preceded the maximum heart rate. Furthermore, the heart rate oscillations were not purely sinusoidal but had a more complex form.

\section{Discussion}

Although several previous studies have exam- ined alterations in ventilatory dynamics at high altitude, ${ }^{12-15}$ little attention has been paid to alterations in heart rate dynamics under these hypoxic conditions. A few previous investigators have commented on cyclical variations in heart rate during sleep at actual ${ }^{13}$ and simulated $^{1617}$ high altitude. However, to our knowledge, the spectral characteristics of this heart rate variability and the possibility of more 

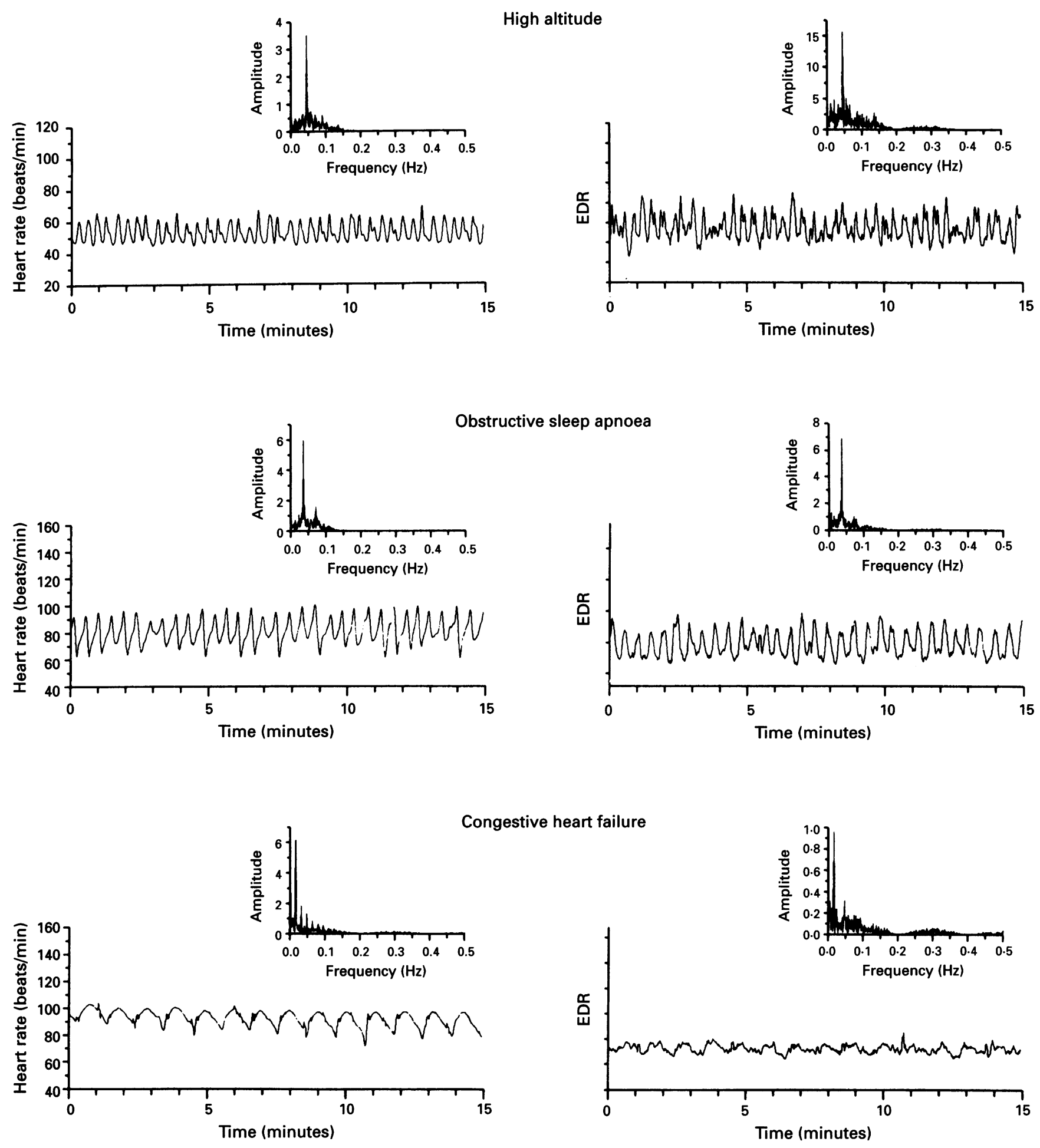

Figure 2 Representative sinus rhythm heart rate-time series (left hand column) and electrocardiographically derived respiratory signals in arbitrary units (EDR; right hand column) both with power spectra shown as inserts in a subject at high alritude, in a patient with obstructive sleep apnoea, and in a patient with congestive heart failure and Cheyne-Stokes breathing. The electrocardiographically derived respiratory signal is low pass filtered using a sliding window average of 10 data points. Note the different cardiopulmonary frequencies in the three clinical conditions. In each condition, however, the heart rate and electrocardiographically derived respiratory power spectra show a spike at the same frequency.

complicated non-linear dynamics have not been fully studied, particularly under actual high altitude conditions. We found the emergence of a wide range of oscillatory heart rate dynamics at high altitude, including periods of prominent low frequency heart rate oscillations at around $0.05 \mathrm{~Hz}$, in eight of the 11 subjects during sleep. These low frequency heart rate oscillations corresponded to alternating cycles of hyperpnoea and apnoea at the same frequency. Therefore, our findings indicate that increased low frequency heart rate power is not simply related to heightened sympathetic tone but to distinctive cardiopulmonary oscillations.

We emphasise that the low frequency heart rate oscillations we observed are distinct from physiological respiratory sinus arrhythmia, which is associated with breath to breath changes in heart rate at the relatively high frequency of about $9-15$ per minute $(0 \cdot 15-0 \cdot 25$ $\mathrm{Hz}$ ). Instead, the heart rate oscillations induced by exposure to high altitude represent much lower frequency cycles at about 3 per 


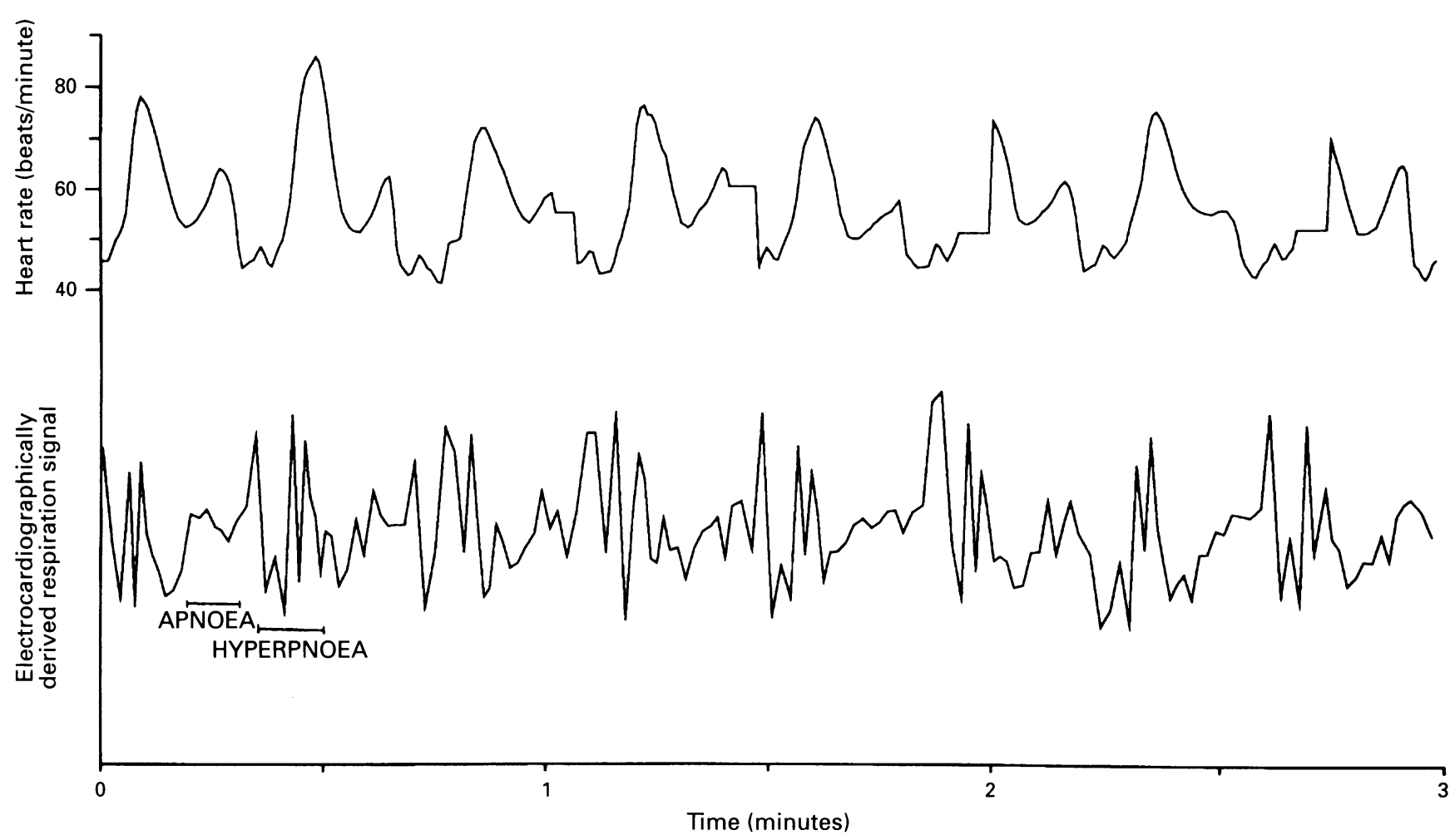

Figure 3 Heart rate over three minutes with the simultaneous electrocardiographically derived respiration signals. Note the complex pattern of sinus rhythm heart rate fluctuations and the alternating periods of hyperpnoea and apnoea in the respiration signal. Periods of hyperpnoea occur just before the peak heart rate.

minute $(0.05 \mathrm{~Hz})$, associated with alternating periods of hyperpnoea and apnoea (figure 3 ). Although normal breathing is by nature periodic, the lower frequency cycles represent an abnormal respiratory pattern conventionally referred to as periodic breathing. During periods of hyperpnoea, breath by breath respiratory cycles at $0 \cdot 1-0 \cdot 2 \mathrm{~Hz}$ are also present (see electrocardiographically derived respiration spectra in figures 2 (top) and 4). The interaction of multiple respiratory cycles at different frequencies is in part responsible for the complex structure of the electrocardiographically derived respiration signal and heart rate time series.

Our finding of increased low frequency heart rate power during the first day of ascent to high altitude is consistent with the observations of Hughson et al in three men during days 4-5 of acclimatisation at $4300 \mathrm{~m} \cdot{ }^{18}$ These investigators found that an increased ratio of low to high frequency power at high altitude was prevented by propranolol, suggesting that it was related to heightened activity of the sympathetic nervous system. Furthermore, the ratio of low to high frequency power decreased by 11-12 days of acclimatisation, possibly because of $\beta$ receptor desensitisation. Unfortunately, they were unable to evaluate the effects of respiration on their findings.

Hughson et al also reported that the ratio of high frequency to total heart rate power (used as an index of parasympathetic nervous system activity) was reduced during days $4-5$ and restored to its sea level value by 11-12 days in three subjects at altitude. ${ }^{18} \mathrm{We}$ did not observe a significant change in the absolute value of high frequency power. If reported as a ratio of high frequency to total heart rate power, our data would also show a decline during early altitude exposure because of an increase in total power. However, our finding that the increase in total power was due primarily to low frequency respiratory oscillations seriously challenges the use of this ratio as a measure of parasympathetic nervous system activity under high altitude conditions.

Our observation of low frequency heart rate and ventilatory oscillations with a cycle time of $20 \mathrm{~s}(0.05 \mathrm{~Hz})$ at $4700 \mathrm{~m}$ agrees remarkably well with findings of other investigators who studied breathing patterns at high altitude. Using a mathematical model of the respiratory control system, Khoo et al predicted the onset of abnormal breathing periodicities with a mean cycle time of about $20 \mathrm{~s}$ at an altitude of 14000 feet (approximately $4267 \mathrm{~m}$ ). ${ }^{15}$ Waggener et al observed periodic breathing with a mean cycle length of $20 \mathrm{~s}$ at $4270 \mathrm{~m},{ }^{19}$ and West et al reported a cycle length of $20.5 \mathrm{~s}$ at $6300 \mathrm{~m} .{ }^{13}$ The phase relation between hyperpnoea and the peak heart rate response illustrated in figure 3 is also similar to that reported by West et al. ${ }^{13}$

Abnormal cyclical breathing patterns are also associated with concordant heart rate oscillations in congestive heart failure with Cheyne-Stokes respiration, ${ }^{45}$ and in the sleep apnoea syndrome. ${ }^{6}$ As illustrated in figure 2, however, the characteristic cardiopulmonary cycle lengths in these two conditions are typically slower than those observed by us and others $^{131719}$ at high altitude. For example, in Cheyne-Stokes respiration the cardiopulmonary cycle length usually ranges from 40 to $65 \mathrm{~s}(0.015-0.025 \mathrm{~Hz}) .{ }^{145}$ This suggests a 
Figure 4 Heart rate and electrocardiographically derived respiration (EDR) autospectra for a representative subject during sleep at high altitude (top). Both show dominant low frequency oscillations at $0.05 \mathrm{~Hz}$. The heart rate and electrocardiographically derived respiration cross spectrum (bottom, left) and heart rate and electrocardiographically derived respiration coherence spectrum (bottom, right) confirm that both signals oscillate at identical frequencies $(0.05 \mathrm{~Hz})$ with a high coherence value of $>0 \cdot 8 .^{11}$ The heart rate and electrocardiographically derived respiration autospectra and cross spectra are normalised to total amplitude.
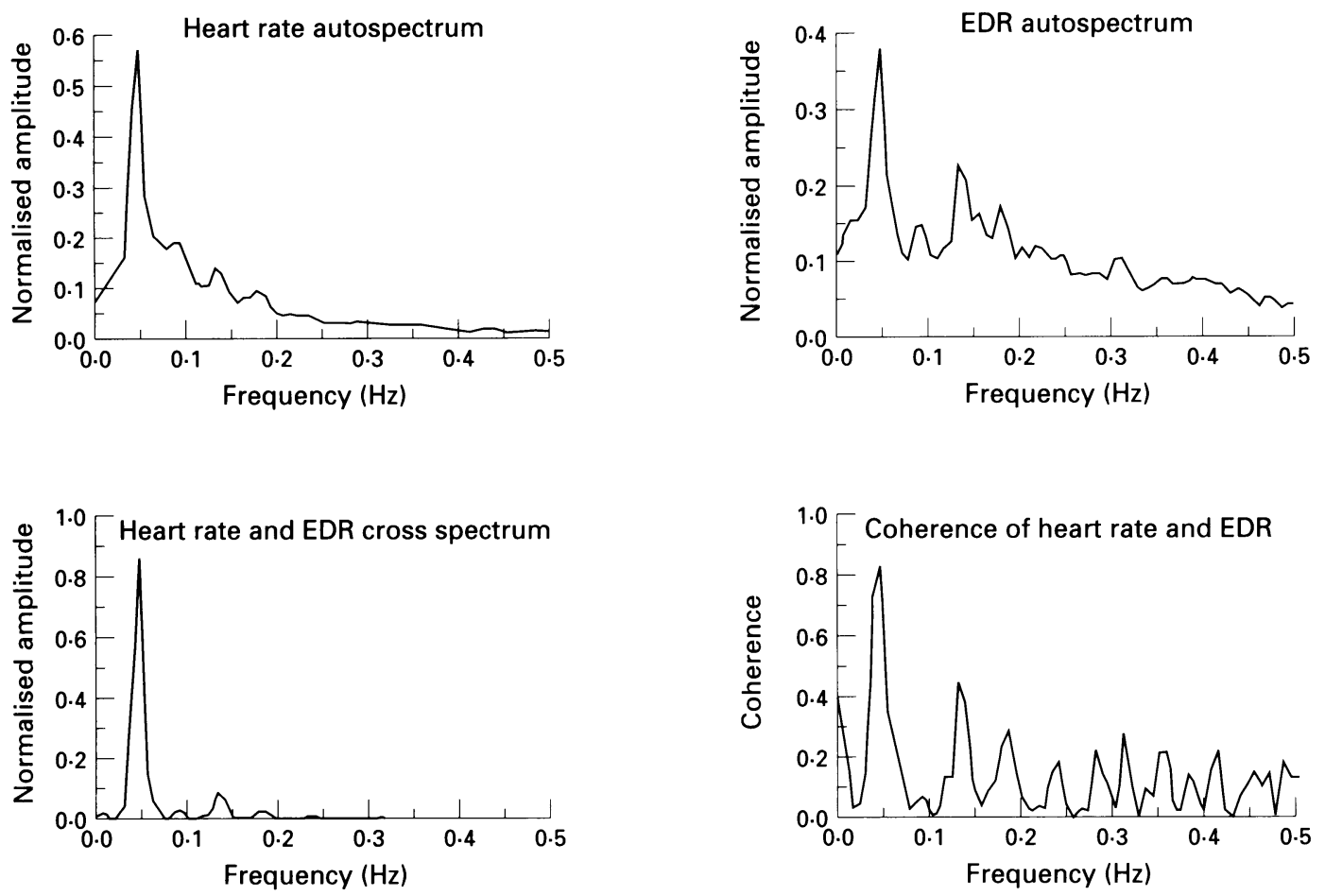

range of pathological cardiopulmonary oscillations reflecting different underlying mechanisms; Cheyne-Stokes breathing presumably results from prolongation of circulatory transit time associated with heart failure ${ }^{20}$ while periodic breathing at high altitude is attributed to increased feedback gain in central or peripheral chemoreceptors due to hypoxaemia. ${ }^{15}$

At various times during the 24 hour heart rate recordings at high altitude we observed the remarkably rich and complicated range of dynamic patterns shown in figure 1. The abrupt changes and switching between states (so called bifurcations) represent non-stationary behaviours that are characteristic of dynamic systems. ${ }^{2321-23}$ Although a precise model for these transitions cannot be inferred solely from the spontaneous cardiopulmonary datasets available in this study, several new biological behaviours are suggested. In particular, abrupt switching between periodic and non-periodic regimens is an anticipated feature of a wide class of non-linear systems displaying one or more of the following: bifurcation behaviour, ${ }^{23}$ intermittency, ${ }^{20}$ stochastic resonance, ${ }^{22}$ or phase transitions (critical phenomena). ${ }^{23}$ We applied these concepts derived from non-linear dynamics and statistical physics to our data because the time series were complex and not explained fully by traditional models.

During periods of wakefulness and extreme exertion non-stationarities in the data represent heart rate responses to physical perturbations, emotional stresses, and environmental changes. Unfortunately, we could not measure and therefore study these influences in the field. Therefore, we limited our analysis to steady state conditions during sleep. Under these conditions we observed striking low frequency oscillations (figure 2). In one of the first descriptions of periodic heart rate changes at high altitude, West et al described a similar saw toothed pattern of heart rate variability. ${ }^{13}$ Our results extend their observation and we have used spectral analysis techniques to confirm the relation between heart rate and pulmonary oscillations at high altitude.

The results of our study are important for several reasons. Firstly, the oscillatory heart rate patterns we observed indicate an underlying non-linear mechanism. ${ }^{1-317}$

Secondly, the patterns are consistent with a highly unstable cardiopulmonary control system that may develop on acute exposure to high altitude before acclimatisation. ${ }^{13} 24$

Thirdly, future attempts to model the physiological mechanisms underlying cardiopulmonary control should account for different types of periodic patterns. For example, any successful "unified" explanation of cardiopulmonary oscillations in different pathological states will need to account explicitly for their differential cycle lengths as shown in figure 2 .

Fourthly, our analysis shows the use of a respiratory signal derived solely from ambulatory electrocardiograms, but independent of the respiratory sinus arrhythmia (electrocardiographically derived respiration) in assessing the interactions between ventilatory and cardiac dynamics. ${ }^{9} 10$

Finally, our data challenge the widespread assumption that low frequency heart rate spectral power measures the activity of the sympathetic nervous system, while high frequency oscillations represent only parasympathetic tone. ${ }^{172526}$ Instead, our results show that an increase in low frequency heart rate power at high altitude is not simply due to a general increase in the $0 \cdot 01-0 \cdot 15 \mathrm{~Hz}$ frequency band but relates specifically to the emergence of a distinct class of cardiopulmonary oscillations associated with a narrow spectral peak (often with higher harmonics). The observation that atropine blunts the low frequency cyclical variation in heart rate associated with the sleep 
apnoea syndrome ${ }^{6}$ also indicates that an increase in low frequency heart rate power is not necessarily attributable solely to heightened sympathetic tone.

Our study has several limitations. Unfortunately, we were unable to study heart rate dynamics after acclimatisation at high altitude. Furthermore, the electrocardiographically derived respiration signal did not permit the measurement of respiratory amplitude and the calculation of transfer functions between respiration and heart rate. The identification of periodic breathing in seven out of eight subjects with low frequency heart rate oscillations may reflect the limited sensitivity of this technique.

Because of the abrupt onset and end of the oscillatory events and the non-stationarity of the data, methods derived from non-linear dynamics-for example, Lyapunov exponent calculation and $1 / \mathrm{f}$ modelling-were not applicable. Future studies of the complex dynamics of heart rate oscillations are needed to elucidate more fully the non-linear mechanisms of cardiopulmonary control, as well as to provide new tools to diagnose pathological breathing states.

This work was supported in part by the Hebrew Rehabilitation Center for the Aged, Boston, Massachusetts; by a National Institute on Aging Teaching Nursing Home Award (AG04390) and by a Claude Pepper Geriatric Research and Training Center Grant (AG08812) from the US Public Health Service, Bethesda, Maryland; by awards from the National Institute on Drug Abuse (P01-DA06316), the National Heart, Lung, and Blood Institute (R01-HL-42172), the National Aeronautics and Space Administration (NAG9-572), and the G Harold and Leila Y Mathers Charitable Foundation; by the Clinical Research Center at the University of New Mexico School of Medicine; by CardioData Corporation; and by the Instituto C Mondino, University of Pavia, Italy.

1 Goldberger AL, Findley LJ, Blackburn RM, Mandell AJ Nonlinear dynamics in heart failure: implications of longwavelength cardiopulmonary oscillations. Am Heart $\mathcal{f}$ 1984;107:612-5.

2 Glass L, Mackey MC. From clocks to chaos: the rhythms of life. Princeton, NJ: Princeton University Press, 1988.

3 Goldberger AL, Rigney DR, Mietus J, Antman EM, Greenwald S. Nonlinear mechanisms in sudden cardiac death syndrome: heart rate oscillations and bifurcations. Experientia 1988;44:983-7.

4 Ichimaru Y, Yanaga T. Frequency characteristics of the heart rate variability produced by Cheyne-Stokes respiration during 24-hr ambulatory electrocardiographic monitoring. Comput Biomed Res 1989;22:225-33.

5 Saul JP, Arai Y, Berger RD, Lilly LS, Colucci WS, Cohen RJ. Assessment of autonomic regulation in chronic congestive heart failure by heart rate spectral analysis. $A m \mathcal{F}$ Cardiol 1988;61:1292-9.
6 Guilleminault C, Connolly S, Winkle R, Melvin K, Tilkian A. Cyclical variation of the heart rate in sleep apnoea syndrome. Mechanisms and usefulness of 24 hour electrocardiography as a screening technique. Lancet 1984; 126-31.

7 Hashimoto F, Appenzeller O, Abrams J, Qualls C. Ambulatory electrocardiographic monitoring at high Ambulatory electrocardiographic mor

8 Lipsitz LA, Mietus J, Moody GB, Goldberger AL. Spectral characteristics of heart rate variability before and during postural tilt. Relations to aging and risk of syncope. Circulation 1990;81:1803-10

9 Moody G, Mark R, Zoccola A, Mantero S. Derivation of respiratory signals from multi-lead ECGs. Computers in Cardiology 1985;12:113-6.

10 Moody GB, Mark RG, Bump MA, Weinstein JS, Berman $\mathrm{AD}$, Mietus JE, et al. Clinical validation of the ECGderived respiration (EDR) technique. Computers in Cardiology 1986;13:507-10.

11 Challis RE, Kitney RI. Biomedical signal processing (in four parts). Part 3. The power spectrum and coherence function. Med Biol Eng Comput 1991;29:225-41.

12 Schoene RB. Control of ventilation in climbers to extreme altitude. Fournal of Applied Physiology, Respiratory, Enviromment and Exercise Physiology 1982;53:886-90.

13 West JB, Peters RM, Aksnes G, Maret KH, Milledge JS, Schoene RB. Nocturnal periodic breathing at altitudes of 6,300 and 8,050 m. F Appl Physiol 1986;61:280-7.

14 Lahiri S, Data PG. Chemosensitivity and regulation of ventilation during sleep at high altitudes. Int $\mathcal{f}$ Sports Med 1992;13:S31-3.

15 Khoo MCK, Kronauer RE, Strohl KP, Slutsky AS. Factors inducing periodic breathing in humans: a general model. fournal of Applied Physiology, Respiratory, Environmental and Exercise Physiology 1982;53:644-59.

16 Malconian $M$, Hultgren $H$, Nitta $M$, Anholm J, Houston $\mathrm{C}$, Fails $\mathrm{H}$. The sleep electrocardiogram at extreme altitude (operation Everest II). Am $\mathcal{f}$ Cardiol 1990;65: $1014-20$

17 Yamamoto Y, Hughson RL, Sutton JR, Houston CS, Cymerman A, Fallen EL, et al. Operation Everest II: an indication of deterministic chaos in human heart rate variability at simulated extreme altitude. Biol Cyberm 1993;69:205-12.

18 Hughson RL, Yamamoto Y, McCullough RE, Sutton JR, Reeves JT. Sympathetic and parasympathetic indicators of heart rate control at altitude studied by spectral analysis. F Appl Physiol 1994;77:2537-42.

19 Waggener TB, Brusil PJ, Kronauer RE, Gabel RA, Inbar $\mathrm{GF}$. Strength and cycle time of high altitude ventilatory patterns in unacclimatized humans. F Appl Physiol 1984; 56:576-81.

20 Guyton AC, Crowell JW, Moore JW. Basic oscillating mechanism of Cheyne-Stokes breathing. Am $\mathcal{F}$ Physiol 1956;187:395-8.

21 Moon FC. Chaotic and fractal dynamics: an introduction for applied scientists and engineers. Wiley: New York, 1992.

22 Gammaitoni L, Menichella-Saetta E, Santucci S Marchesoni F, Presilla C. Periodically modulated bistable systems: stochastic resonance. Phys Rev A 1989, 40:2114-9.

23 Stanley HE, Ostrowsky N, eds. Correlations and connectivity: geometric aspects of physics, chemistry and biology. Boston Kluwer Academic, 1990

24 Pack AI, Gottschalk A. Mechanisms of ventilatory periodicities. Ann Biomed Eng 1993;21:537-44.

25 Pagani M, Lombardi F, Guzzetti S, Rimoldi O, Furlan $R$ Pizzinelli $\mathrm{P}$, et al. Power spectral analysis of heart rate and arterial pressure variabilities as a marker of sympathovagal interaction in man and conscious dog. Circ Res 1986;59:178-93.

26 Pagani M, Lombardi F, Malliani A. Heart rate variability: disagreement on the markers of sympathetic and parasympathetic activities. $\mathcal{F}$ Am Coll Cardiol 1993;22: parasym. 